\title{
Strong bonding of corneal incisions using a noncontact fiber-optic laser soldering method
}

Svetlana Basov

David Varssano

Max Platkov

Ilan Gabay

Mordechai Rosner

Irina Barequet

Marcel Rattunde

Joachim Wagner

Mickey Harlev

Doron Ofer

Ilana Nisky

Yair Dankner

Abraham Katzir 


\title{
Strong bonding of corneal incisions using a noncontact fiber-optic laser soldering method
}

\author{
Svetlana Basov, ${ }^{a}$ David Varssano, ${ }^{\mathrm{b}}$ Max Platkov ${ }^{\mathrm{c}, \star}$ Ilan Gabay, ${ }^{\mathrm{d}}$ Mordechai Rosner, ${ }^{\mathrm{e}}$ Irina Barequet, ${ }^{\mathrm{e}}$ \\ Marcel Rattunde, ${ }^{f}$ Joachim Wagner, ${ }^{f}$ Mickey Harlev, ${ }^{\mathrm{g}}$ Doron Ofer, ${ }^{\mathrm{h}}$ Ilana Nisky, ${ }^{\mathrm{i}}$ Yair Dankner, ${ }^{\mathrm{j}}$ and \\ Abraham Katzir ${ }^{\mathrm{d}}$ \\ ${ }^{a}$ Tel-Aviv University, Department of Biomedical Engineering, Tel Aviv, Israel \\ ${ }^{\mathrm{b} T e l-A v i v}$ University, Tel-Aviv Sourasky Medical Center and Sackler Faculty of Medicine, Department of Ophthalmology, Tel-Aviv, Israel \\ 'Nuclear Research Center Negev, Beer-Sheva, Israel \\ ${ }^{\mathrm{d} T e l-A v i v}$ University, School of Physics and Astronomy, Tel-Aviv, Israel \\ 'Tel Aviv University, Goldschleger Eye Institute, Sheba Medical Center, Ramat Gan, and Sackler Faculty of Medicine, Tel-Aviv, Israel \\ ${ }^{\mathrm{f}}$ Fraunhofer-Institut für Angewandte Festkörperphysik, Freiburg, Germany \\ ${ }^{9} T e l-A v i v$ University, Veterinary Service Center, Sackler Faculty of Medicine, Tel-Aviv, Israel \\ hLahav Research Institute, Kibbutz Lahav, D.N. Negev, Israel \\ 'Ben-Gurion University of the Negev, Department of Biomedical Engineering, Beer-Sheva, Israel \\ ishenkar College of Engineering and Design, Ramat-Gan, Israel
}

\begin{abstract}
Suturing of corneal incisions requires significant skill. We demonstrate a noncontact method that will simplify the bonding process. 5-mm-long penetrating vertical and slanted incisions were made in corneas of eyes, extracted from dead piglets. A fiber-optic laser system was used for laser soldering of the incisions, under close temperature control, using albumin solder. The burst-pressure $P_{\mathrm{B}}$ immediately after the soldering was found to be $P_{\mathrm{B}} \approx 92$ and $875 \mathrm{mmHg}$, for vertical and slanted incisions, respectively. $P_{\mathrm{B}}=875 \mathrm{mmHg}$ is an exceptionally high figure, $\approx 10$ times the clinically acceptable value for sutured incisions. Laser soldering was then performed on penetrating incisions made in the corneas of live healthy piglets, of weight $\approx 10 \mathrm{Kg}$. After a healing period, the eyes were extracted, and the corneas were examined by histopathology and by optical coherence tomography. Our method immediately generated watertight and strong bonding without noticeable corneal shape distortion. These results would be beneficial for cataract surgery and for corneal transplantations. The fiber-optic system makes it much easier to bond corneal incisions. In the future, laser soldering could be automated and efficiently used by less experienced surgeons, thereby reducing the workload on the experienced ones. () The Authors. Published by SPIE under a Creative Commons Attribution 4.0 Unported License. Distribution or reproduction of this work in whole or in part requires full attribution of the original publication, including its DOI. [DOI: 10.1117/1.JBO.24.12.128002]
\end{abstract}

Keywords: infrared; laser soldering; laser bonding; albumin; cornea; optical fibers.

Paper 190279RR received Aug. 21, 2019; accepted for publication Nov. 11, 2019; published online Dec. 28, 2019.

\section{Introduction}

The closure of incisions in tissues involves passing a needle through the tissues, close to the edges, pulling them together and relying on tissue and suture strength to hold them tight. Suturing is a delicate, demanding, and time-consuming procedure that requires significant technical skill. ${ }^{1,2}$ Sutured sites have an increased risk for leaks, infections, and scarring. ${ }^{3,4}$

Laser-tissue bonding has been introduced in an attempt to solve these problems. There are two methods of laser-bonding of incisions, ${ }^{5-7}$ which differ by their biochemical mechanisms: photothermal bonding and photochemical bonding.

(A) Photothermal bonding. There are two basic photothermal procedures: laser welding and laser soldering. In laser-welding, a spot on the approximated edges of an incision is heated by laser light absorption in the tissue or in a coloring agent (which changes the penetration depth of the light) ${ }^{8-10}$ The spot is heated to temperature $T$ for time $t$, causing adhesion. The full length of the incision is then bonded, spot by spot. In laser-soldering, a biological bonding agent, such as, albumin or chitosan, ${ }^{11,12}$ is applied at the approximated edges and heated spot by spot. The heating denatures proteins and links them with collagen, thus generating a clot that seals the incision. ${ }^{13}$

*Address all correspondence to Max Platkov, E-mail: mmaaxxpp@gmail.com
Laser bonding, and specifically the introduction of a biological agent (e.g., albumin), generates a watertight seal and accelerates healing with very little scarring. In addition, it improves the immediate mechanical properties (e.g., tensile strength) of the target tissues after bonding. During the last few years, there has been significant progress in the development of laser bonding methods. Photothermal bonding has already been demonstrated in animal $^{14,15}$ and clinical ${ }^{9}$ works.

(B) Photochemical bonding. A chemical dye that is applied at the incision is sensitized by visible laser light. The irradiation drives a chemical reaction that initiates bonding through protein crosslinking. ${ }^{16-18}$ With this approach, significant bond strengths have been achieved in many tissues. Photochemical bonding has also been demonstrated in animal experiments (including ones on the cornea) and clinically. ${ }^{16,19}$

These two bonding methods have a potential of being widely used clinically. But suturing is obviously the standard method still used for closing incisions.

Our analysis of previous works showed that many researchers calculated the heat distribution generated by laser heating, under various experimental protocols, using different lasers, heating setups, and solder compounds. ${ }^{20-22}$ However, very little work was done on monitoring and controlling the temperature of each heated spot on the incision. 
The lack of temperature control caused underheating or overheating, and in both cases, the bonding strength was insufficient. Attempts have been made in the past to develop such a control, but more work is still needed. One pioneering work had a camera adapted for temperature-control of laser bonding and was successfully used clinically. ${ }^{9,14}$ Yet, none of these approaches has been widely used.

We developed a fiber-optic system that used a middleinfrared (mid-IR) detector to measure the temperature $(T)$ of the heated spot, during the irradiation time $(t)$. A feedback loop was used to control the laser output so that $T$ was kept constant. We concentrated on laser soldering and used albumin as a solder. In addition to the experiments, we also carried out theoretical calculations of the temperature distribution during irradiation. ${ }^{20,23-27}$ We tested our calculations at different values of $T$ and $t$ and found optimal conditions for $T \approx 60^{\circ} \mathrm{C}$ and $t \approx 10 \mathrm{~s}$. This was also verified experimentally, and strong and watertight bonding, without noticeable thermal damage, was obtained. $^{28}$

Using this information, we succeeded in soldering incisions in vitro in skin, ${ }^{29}$ bowel, ${ }^{30} \mathrm{dura},{ }^{31}$ and trachea. ${ }^{32}$ In experiments on skins of large farm pigs in vivo, the wound healing was faster than that obtained after standard suturing, and there were practically no scars. ${ }^{33}$ Clinical laser-soldering protocols were also carried out on incisions in the abdomen skins of patients, after laparoscopic cholecystectomy. Again, strong bonding with very little scarring was obtained. ${ }^{24,34}$

Recently, we used robotic laser soldering for bonding incisions in mouse skins in vitro and obtained a high bond strength. ${ }^{35}$ Using this technique, we were able to deliver a measured amount of heat for a predefined amount of time, repeatably, thereby obtaining standardization and allowing for remote (e.g., daVinci System) operation or even an unaided one.

Suturing is a standard procedure in cataract treatment, corneal surgery, or corneal transplantation (penetrating keratoplasty). It is inexpensive, reliable, and readily available. ${ }^{3}$ However, the tissue is very delicate and presents a considerable challenge. One complication in corneal transplantation is astigmatism, due to nonuniform tensions generated by the sutures around the cut, resulting in corneal deformation. ${ }^{4}$ Chemical adhesives have also been used in corneal transplantation, but they can have inadequate tensile strength, they are toxic, and suffer from other problems. ${ }^{36,37}$

In our previous experiments on laser soldering of corneal incisions in piglets, we proved that our noncontact and temperature-controlled soldering method, based on a $\mathrm{CO}_{2}$-laser, generated an immediate, reproducible, and watertight bond. ${ }^{21,29,38}$

One goal of the current research was to maximize the burst pressure $P_{\mathrm{B}}$ of soldered corneal incisions. The second goal was to check if there is any change in the corneal shape of the piglets due to the soldering process. To fulfill the first goal, we found the optimal conditions needed to generate immediate very high $P_{\mathrm{B}}$ ex vivo. We report here these optimal conditions. To achieve the second goal, we used these optimal conditions, assuming that the same $P_{\mathrm{B}}$ will be obtained in live piglets. We allowed the eyes to heal for 6 weeks and observed the corneal shape, using optical coherence tomography (OCT) and histopathology. We did not observe changes in the shape of the cornea as a result of the soldering. Obtaining immediate strong bonding on corneal incisions, without deforming the retina, will be an important step toward bringing laser soldering of corneal incisions from the laboratory to clinical use.

\section{Materials and Methods}

\subsection{Choice of Wavelength}

The choice of laser wavelength that generates strong bonding greatly depends on the penetration depth of the laser radiation into the tissue. In the past, we used a $\mathrm{CO}_{2}$ laser, emitting at $10.6 \mu \mathrm{m}$, for heating spots on the incision. However, the penetration depth at this wavelength is only a few microns, and therefore the bonding of the incisions was not very strong. In this work, we used a semiconductor disk laser emitting 0 to $0.5 \mathrm{~W}$ at $1.9 \mu \mathrm{m}$ (Fraunhofer Institute, Freiburg, Germany). ${ }^{39}$ The radiation at this wavelength is fully absorbed within few hundred microns, so it heated the full thickness of the cornea. We previously established ${ }^{24}$ that this operation generated strong bonding while ensuring no retinal damage.

\subsection{Beam Delivery and Temperature Control}

We developed a fiber-optic laser soldering system with an improved handpiece, combining two types of optical fibers arranged in a bundle. The bundle included a silica fiber, surrounded by 4 to $6 \mathrm{AgClBr}$ fibers that had been made by the Applied Physics Group at Tel-Aviv University ${ }^{24,35}$ and were highly transparent in the mid-IR. The silica fiber transmitted the 1.9- $\mu \mathrm{m}$ laser radiation from the proximal end to the distal end of the bundle to heat a spot of diameter of $\approx 2 \mathrm{~mm}$ on the incision. The heated spot emitted mid-IR black-body radiation that was proportional to temperature $T$. The $\mathrm{AgClBr}$ fibers transmitted the black-body radiation, from their distal ends to their proximal ends. A mid-IR detector (Infratec LME-335) was connected to the proximal ends, and it detected the radiation and generated voltage $V$, which was also proportional to the spot $T$. $V$ was used in a feedback loop to control the laser intensity, keeping $T$ constant. The speed of update of the laser intensity was $\sim 200 \mathrm{~Hz}$. The optimal conditions for laser bonding had already been determined in previous studies and were $55<T<65^{\circ} \mathrm{C}$ for $8<t \leq 12 .^{20,28}$ In this study, we used only these values.

\subsection{Solder Preparation}

The solder was prepared from $67 \%$ w/v bovine albumin solution (Sigma Chemicals, St. Louis, Missouri) in doubly distilled water.

\subsection{Surgical Protocols}

By observing the process through a microscope (Fig. 1), the surgeon followed these protocols:

1. A linear incision of length $\approx 5 \mathrm{~mm}$ was made on the cornea. Each incision was made in one of two modes: (I) perpendicular to the corneal surface [Fig. 2(A1); this cut was used only in the ex vivo experiments], or (II) slanted with respect to the corneal surface [Fig. 2(A2); this cut was used both in the in vivo and the ex vivo experiments].

2. Only for the laser-soldering cases: a layer of solder of thickness $\approx 500 \mu \mathrm{m}$ was spread over the approximated edges of the incision and some solder was spread between the edges. 


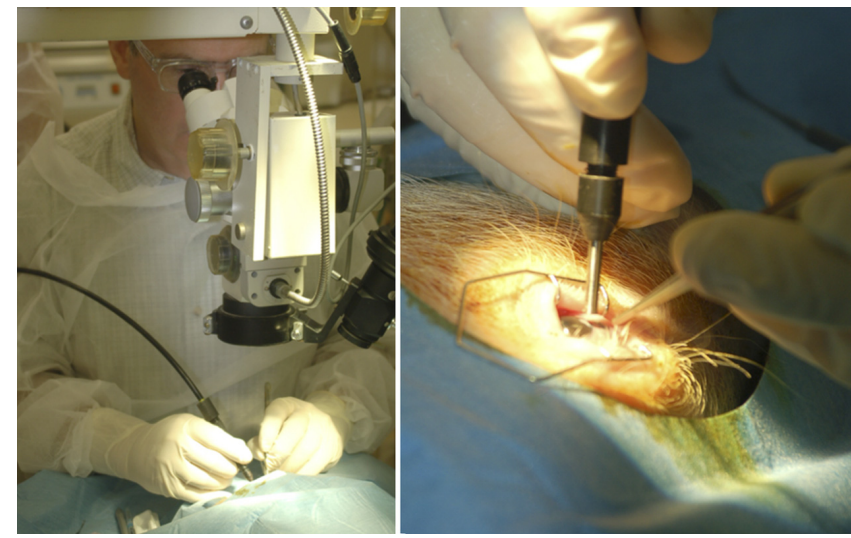

Fig. 1 The laser soldering protocol, using the laser soldering system.
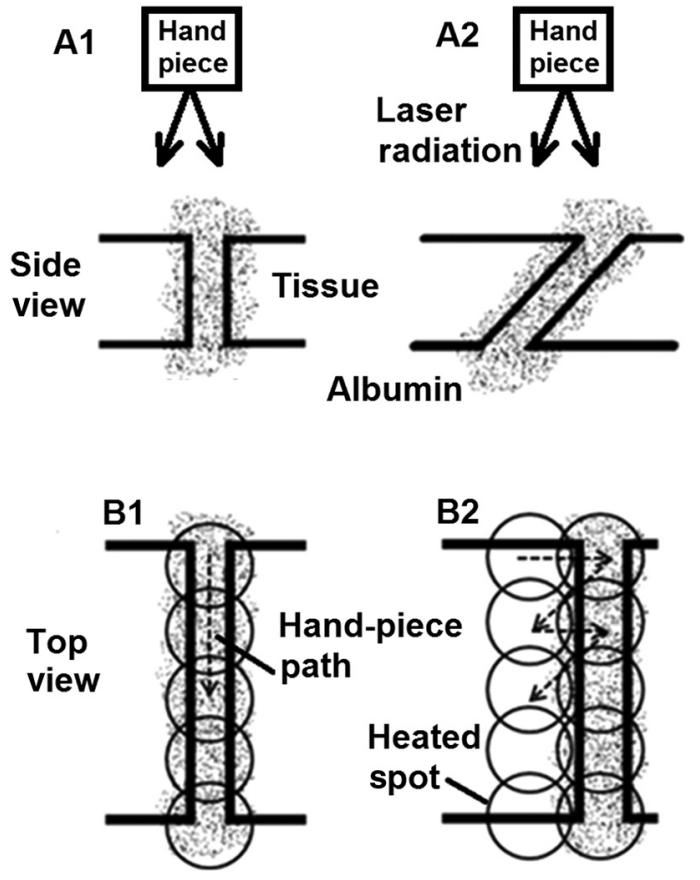

Fig. 2 Soldering protocols: (A1) Cross section of a perpendicular (vertical) incision. (A2) Cross section of a slanted incision. (B1) Top view of a linear heating along an incision. (B2) Top view of a zigzagpattern heating along an incision. The dotted area marks the places where albumin was applied. The circles mark the heated-spot diameter. The broken arrows mark the direction of the heating spot by spot. This figure is not in scale, the cut edges have been moved far apart, for clarity.

3. The distal tip of the handpiece was brought to a height 3 to $5 \mathrm{~mm}$ above the incision, and the laser beam heated a spot on the incision to the optimal values of $T$ and $t$. The tip was then moved to a neighboring spot along the incision, according to one of two patterns: (I) linear heating [Fig. 2(B1)] or (II) zigzag heating [Fig. 2(B2)]. During our experiments, we used for example a vertical incision-zigzag heating protocol or a slanted incision-linear heating protocol. In most cases, we used a slanted incision-zigzag heating protocol.

\subsection{Ex Vivo Bonding}

The first part of the research consisted of ex vivo experiments on eyes, freshly extracted from dead piglets $(<24 \mathrm{~h})$. Linear incisions were made in each of these corneas, and the incisions were then laser soldered. We checked the condition for obtaining optimal $P_{\mathrm{B}}$. These included the optimal laser wavelength, the irradiation temperature $T$, the time $t$, and the surgical protocol itself. The optimal conditions were then used for in vivo experiments on live piglets.

\subsection{In Vivo Bonding}

The ex vivo protocol (Sec. 2.4) that generated the best $P_{\mathrm{B}}$ was selected for the in vivo application. The piglets were divided into three groups. In the laser soldering group, the albumin solder was spread both inside and over the incisions, before the heating. In the laser welding, no solder was used. In both cases, the edges of each incision were approximated, and laser bonding was performed under close temperature control. The piglet-eyes of the third, control group, were incised, and left to self-seal and self-heal.

\subsection{Testing the Quality of the Bonding and the Corneal Shape}

In this work, we did not test the thermal damage. However, in previous studies on porcine corneas, we observed that none of the features that characterize damaged tissues (e.g.,, different degrees of coagulation) was visible by histologic or microscopic observations. $^{6,40}$

The following methods were used to assess the quality of the bonded incisions in the corneas.

1. Burst pressure $\left(P_{\mathrm{B}}\right)$ : Water was introduced into each eye through a hypodermic needle and the water pressure was gradually increased till, at some pressure, a leak was detected somewhere on the organ. This pressure was then logged and called $P_{\mathrm{B}}$. This $P_{\mathrm{B}}$ was measured by a Lutron PS9302 pressure transducer.

2. OCT: The corneas with bonded incisions were scanned with an OCT apparatus (Visante OCT, Carl Zeiss Meditec, Jena Germany). ${ }^{27}$ This method was also used to evaluate the radii of curvature of the corneas, and to compare the results with ones obtained for corneas of eyes that had not been used in our experiments.

3. Histopathology: The same corneal samples were used for histopathological evaluation. The eyes were fixed in formalin $4 \%$, embedded in paraffin, and sectioned through the soldered incision area. Hematoxylin and eosin (H\&E) staining was applied before evaluating them by light microscopy.

\subsection{Animal Treatment}

\subsubsection{Before surgery}

Twelve piglets were grown in the Lahav Research Institute (LRI), each weighing $\approx 10 \mathrm{Kg}$. They were anesthetized by an intramuscular ketamine and xylazine mixture $0.1 \mathrm{ml} / 100 \mathrm{~g}$ at $3: 4$ ratio. Afterward, 5\% polydine solution was spread on the ocular surface and the surrounding skin, to sterilize the surgical area. The surgical protocols were then performed, under sterile 
conditions, with the aid of an operating microscope (Inami, Japan). In each piglet, a short, linear incision of length $\approx 5 \mathrm{~mm}$ was made in the cornea of the left eye, near the limbus, and the slanted incision-linear heating protocol was used.

\subsubsection{After surgery}

After the experiments, the piglets were kept at the LRI for 6 weeks, for postsurgery inspection and analysis. Then, they were euthanized and the left eye (the one with the incision) of each piglet was surgically removed and sent to the Tel-Aviv Sourasky Medical Center. There, an OCT system was used to obtain a cross-section image of each of the corneas at the incisions. The eyes were then placed in formaldehyde and sent to the Sheba Medical Center for Histopathology. Our work was performed under the guidelines of the Helsinki Accord, authorization number 01-16-026, the State of Israel, and Tel-Aviv University.

\section{Results}

\subsection{Ex-Vivo Experiments}

Several soldering protocols were tested (Fig. 2) for soldering of incisions on the cornea. The results (Table 1) showed that for the slanted incision-zigzag heating protocol the average $P_{\mathrm{B}}$ for 14 eyes was $(875 \pm 274) \mathrm{mmHg}$. It is an order of magnitude higher than the average value of $(92 \pm 8.4) \mathrm{mmHg}$, obtained for 18 eyes, where the vertical incision-linear heating protocol (Table 1) was used. The latter is also higher than the clinically accepted value of $P_{\mathrm{B}}=80 \mathrm{mmHg}$ for sutured corneal incisions. $^{21,37}$

We also did laser welding of incisions ex vivo: we used either one of our heating protocols, without any solder, as preliminary experiments in preparation for the in vivo experiments. The average $P_{B}$ measurements in these cases (not shown) exhibited bond strengths of $\sim 30 \pm 10 \mathrm{mmHg}$ in 15 porcine eyes, which is far below the acceptable value of $80 \mathrm{mmHg}$.

We compared our results by calculating the one-sample $T$ test of each experiment with the accepted value of $80 \mathrm{mmHg}$ for suturing. We obtained $P$-values that are smaller than $2 \%$, $0.1 \%$, and $0.01 \%$, for the soldering methods 1,2 , and 3 , as shown in Table 1, respectively. Calculating the unpaired twosample $T$-tests for either permutation of the average $P_{\mathrm{B}}$ of any two of these bonding methods, yielded $P$-values $<0.01$. These results are statistically significant.

OCT and histopathology of the soldered incisions in these eyes showed well-approximated tissues, and no noticeable corneal shape distortions at the area of the cut (not shown).

Furthermore, qualitative comparisons of the radii-of-curvature of corneas soldered by our protocols, to those that had been welded, were carried out using OCT and histopathology imaging. They exhibited no noticeable changes in the radii of curvature, suggesting no or minimal generation of optical aberrations to the cornea by the laser soldering protocol. These are not shown for the ex vivo results but are shown representatively for the in vivo results, in Fig. 3.

\subsection{In-Vivo Experiments}

The success in immediately achieving high $P_{\mathrm{B}}$ after soldering in the ex vivo experiments paved the way for obtaining permission for carrying out the in vivo experiments in live piglets. In these, we used the temperature-controlled laser-bonding of corneal incisions. Maintaining the bonding protocols, we considered it safe to assume that $P_{\mathrm{B}}$ values in the in vivo and in the ex vivo experiments, immediately after the surgery, will be similar. Our next stage was to laser-solder incisions in the corneas of live piglets, followed by a postoperative follow-up on the bonded incisions.

Table 1 Burst-pressure $\left(P_{\mathrm{B}}\right)$ measured after ex vivo soldering of incisions of length $\approx(5 \pm 1) \mathrm{mm}$ in porcine corneas, using different soldering protocols.

\begin{tabular}{|c|c|c|c|c|c|c|c|c|}
\hline ID & Bonding method & Eye ID & $P_{\mathrm{B}}(\mathrm{mmHg})$ & Eye ID & $P_{\mathrm{B}}(\mathrm{mmHg})$ & Eye ID & $P_{\mathrm{B}}(\mathrm{mmHg})$ & $\begin{array}{c}P_{\mathrm{B}}(\mathrm{mmHg}) \\
\text { average }\end{array}$ \\
\hline \multirow[t]{3}{*}{1} & $\begin{array}{l}\text { Make a vertical incision, spread albumin, } \\
\text { solder the cut in a linear motion. }\end{array}$ & 1 & 69 & 4 & 100 & 7 & 83 & $89.5 \pm 10.4$ \\
\hline & & 2 & 84 & 5 & 98 & 8 & 96 & \\
\hline & & 3 & 91 & 6 & 95 & & & \\
\hline \multirow[t]{4}{*}{2} & $\begin{array}{l}\text { Make a vertical incision, spread albumin, } \\
\text { and solder the cut in a zigzag motion. }\end{array}$ & 9 & 83 & 13 & 98 & 17 & 97 & $92 \pm 8.4$ \\
\hline & & 10 & 81 & 14 & 81 & 18 & 85 & \\
\hline & & 11 & 103 & 15 & 96 & & & \\
\hline & & 12 & 99 & 16 & 95 & & & \\
\hline \multirow[t]{5}{*}{3} & Make a slanted incision, spread albumin, & 19 & 1025 & 24 & 1350 & 29 & 626 & $875 \pm 274$ \\
\hline & & 20 & 1024 & 25 & 650 & 30 & 800 & \\
\hline & & 21 & 1020 & 26 & 420 & 31 & 660 & \\
\hline & & 22 & 1130 & 27 & 644 & 32 & 846 & \\
\hline & & 23 & 1300 & 28 & 754 & & & \\
\hline
\end{tabular}




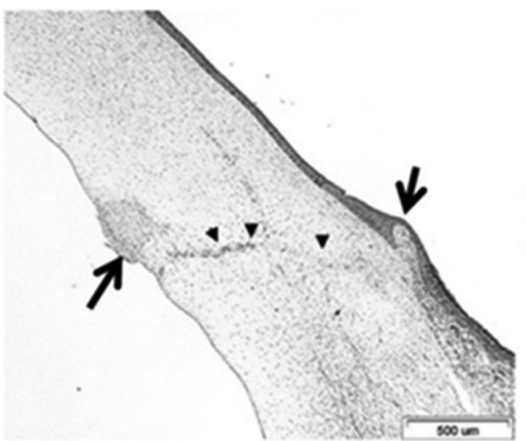

Piglet D, Soldering
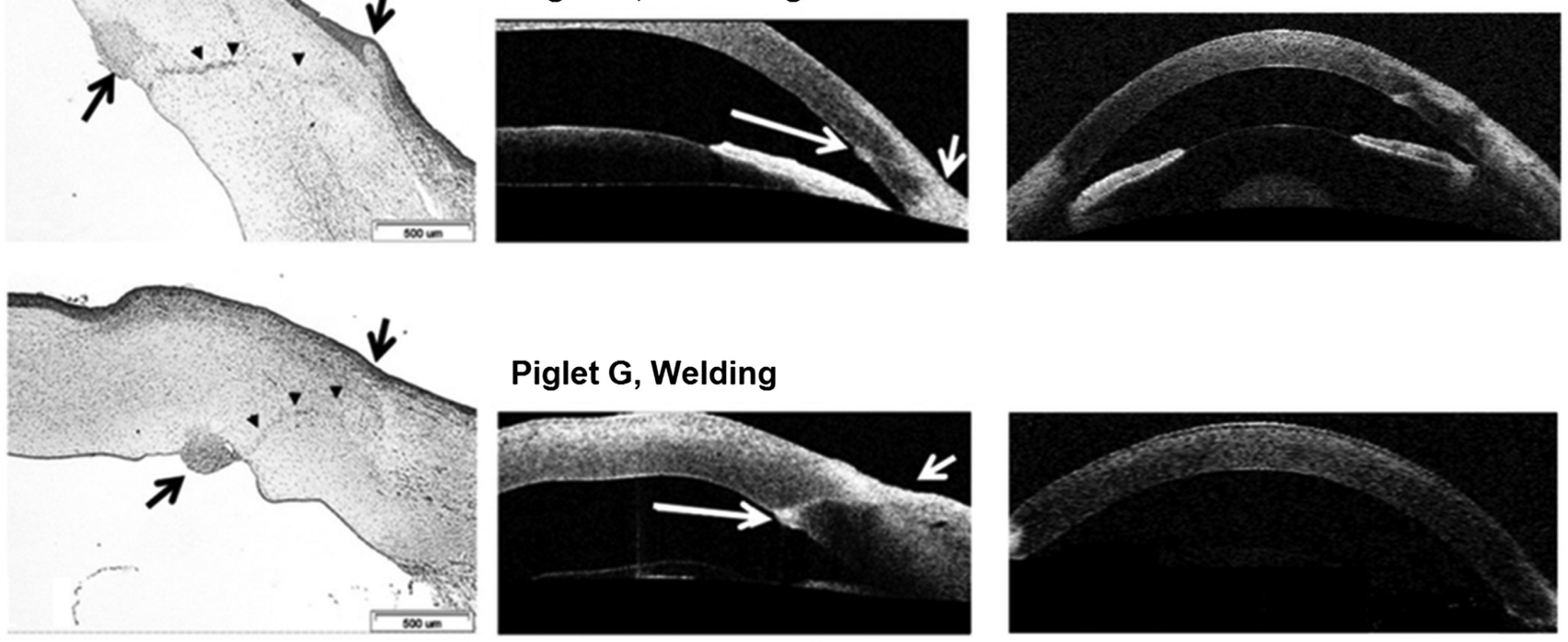

\section{Piglet G, Welding}
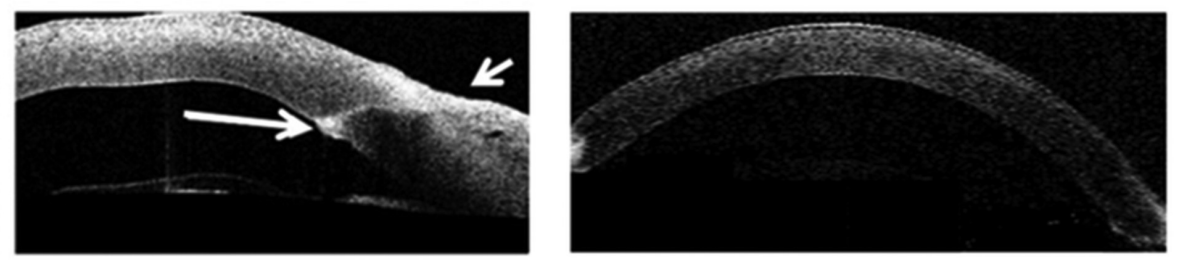

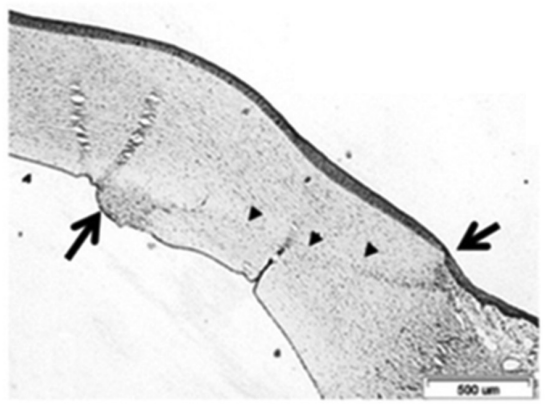

(a)

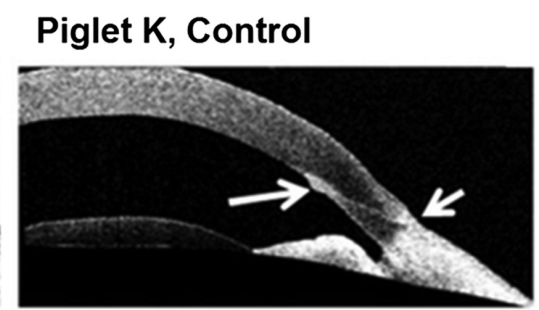

(b)

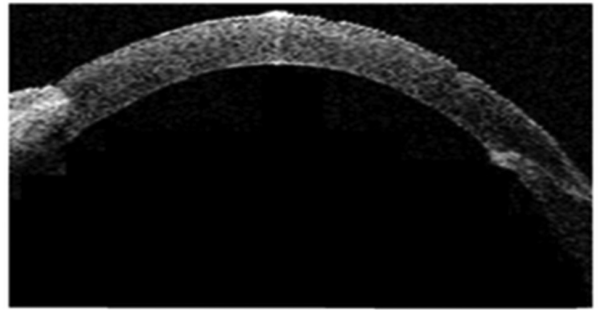

(c)

Fig. 3 Each row shows three images of a cross section of a bonded incision: (a) histopathology, (b) OCT at high magnification, and (c) OCT at low magnification. The top row represents a soldered incision in the cornea of piglet-D (Table 2). It was soldered using the slanted incision-zigzag heating protocol. The center row represents welded incision in the cornea of piglet-G (Table 2). It was welded using the slanted incision-zigzag heating protocol. The bottom line represents the cornea of piglet-K (Table 2). This piglet belonged to the control group where a slanted incision was not laser heated.

The aim of the follow-up was the observation of the corneal shape. It was also safe to assume that after a recovery period of 6 weeks, the major contribution to $P_{\mathrm{B}}$ should have been attributed to the recovery, rather than to the protocol. Therefore, $P_{\mathrm{B}}$ was not tested on in vivo eyes.

The results are presented in Table 2 and summarized in Fig. 3. Three OCT findings and six histology findings are compared, among the piglet-eyes that were operated on. The OCT parameters are: (1) the observation of the slanted cut, (2) the extent of the hyperreflectivity on the anterior corneal side, and (3) the extent of the hyperreflectivity of the posterior stromal side. The histology parameters are: (1) the bonding quality, (2) the extent of epithelium thinning, (3) the folding of the basal-cell layer due to the heating, (4) the appearance of scarring, (5) the hypercellularity and neo-vascularization, and (6) the integrity of the Descemet's membrane. The first row in Table 2, describing the results for piglet, A, lists these findings, in a numbered order. The other piglets refer to these findings in the same order, with the appropriate changes.
In Fig. 3, we describe the typical OCT and histopathological results of three bonded incisions in three piglet corneas. Each cornea represented one of the three groups: soldered corneas, welded corneas, and a control group. Each incision is marked by two arrows.

These images show that the incisions from the epithelium to the endothelium (the two bold arrows marking the incision) were made at the edge of each cornea, near the limbus. They mark the entry and exit points at the front and back corneal surfaces (as opposed to depth, which is measured at a right angle to the corneal surface). The arrowheads mark the location of the disruption. This is the stromal scar from the epithelium to the endothelium, the dark line in the histological images, or the bright line in the OCT images.

\subsubsection{Soldered group}

The incision shown in Fig. 3 for piglet D was soldered using the slanted incision-zigzag heating protocol. A well-demarcated 
scar is clearly seen. This image shows good bonding, closed incision, and intact corneal shape, which indicates that no changes in corneal shape were caused by the soldering protocol.

The OCT analyses revealed slanted prolonged penetrating incisions, with hyperreflective anterior stroma, encompassing roughly the outer $40 \%$ to $70 \%$ of the incision length. A small area of the posterior stromal hyperreflectivity, near the endothelial side of the incision is seen. In one piglet, we could detect a prolonged area of the posterior stromal hyperreflectivity, near the endothelial side of the wound. In another piglet, we found an area of posterior stromal hyperreflectivity, near the endothelial side of the wound that bulged into the anterior chamber.

The histopathology analyses of the white-light microscopic images of the corneas in this group revealed well-bonded incisions in the corneal tissues. In the area of the bonded cut, the epithelium was mildly thinned, and the basal cell layer showed

Table 2 Experimental results for laser-soldering of piglet corneal-incisions, in vivo.

\begin{tabular}{|c|c|c|c|}
\hline Action & Piglet & OCT & Histology \\
\hline \multirow[t]{4}{*}{$\begin{array}{l}\text { Group I solder: } \\
\text { slanted incision, } \\
\text { zigzag heating }\end{array}$} & A & $\begin{array}{l}\text { 1. Slanted prolonged penetrating cut. } \\
\text { 2. Hyperreflective anterior corneal side, encompassing } \\
\text { roughly the outer } 40 \% \text { to } 70 \% \text { of the wound length from } \\
\text { the entry at the front corneal surface to the exit at the } \\
\text { back corneal surface. } \\
\text { 3. Small area of the posterior stromal hyperreflectivity } \\
\text { near the endothelial side of the wound. }\end{array}$ & $\begin{array}{l}\text { 1. Well-bonded corneal cut. } \\
\text { 2. Mildly thinned epithelium at the bonded cut. } \\
\text { 3. The basal cell layer shows mild folding. } \\
\text { 4. Thin epithelium-to-endothelium stromal scar. } \\
\text { 5. The scar shows mild hypercellularity and } \\
\text { neovascularization. } \\
6 \text {. The Descemet's membrane is disrupted and } \\
\text { the gap between its edges is occupied by } \\
\text { a triangular scarring with the base toward the inner } \\
\text { surface of the cornea. }\end{array}$ \\
\hline & $\mathrm{B}$ & $\begin{array}{l}\text { 1. Same as the OCT results for piglet } A \text {. } \\
\text { 2. Same as piglet A but only } 50 \% \text { of the wound is } \\
\text { encompassed. } \\
\text { 3. Prolonged posterior stromal hyperreflective area near } \\
\text { the wound endothelium. }\end{array}$ & Same as the histology results for piglet $A$. \\
\hline & C & $\begin{array}{l}\text { 1, 3. Same as the OCT results for piglet } A \text {. } \\
\text { 2. } 40 \% \text {. }\end{array}$ & Same as the histology results for piglet $A$. \\
\hline & D & $\begin{array}{l}\text { 1. Same as the OCT results for piglet } A \text {. } \\
\text { 2. } 70 \% \text {. } \\
\text { 3. Area of the posterior stromal hyperreflectivity near } \\
\text { the endothelial side of the wound, bulging into the } \\
\text { anterior chamber. }\end{array}$ & $\begin{array}{l}1,2,3,4 \text {. Same as the histology for piglet } A \text {. } \\
\text { 5. The scar shows mild hypercellularity and } \\
\text { extensive neovascularization. } \\
6 . \text { The Descemet's membrane is disrupted and } \\
\text { is covered by endothelium. }\end{array}$ \\
\hline \multirow[t]{4}{*}{$\begin{array}{l}\text { Group-II weld: } \\
\text { no albumin, } \\
\text { slanted incision, } \\
\text { zigzag heating }\end{array}$} & $E$ & $\begin{array}{l}\text { 1, 2. Same as the OCT results for piglet } D \text {. } \\
\text { 3. Minimal posterior stromal hyperreflectivity near } \\
\text { the wound endothelium within some localized stromal } \\
\text { thinning. }\end{array}$ & Same as the histology results for piglet D. \\
\hline & $\mathrm{F}$ & $1,2,3$. Same as the OCT results for piglet $D$. & Same as histology results for piglet D. \\
\hline & G & $\begin{array}{l}\text { 1, 3. Same as OCT results for piglet } D \text {. } \\
\text { 2. } 50 \% \text { to } 70 \% \text {. }\end{array}$ & $\begin{array}{l}1,2,4,5,6 \text {. Similar to the histology for piglet } A \text {. } \\
\text { 3. Not seen. } \\
\text { Extensive inflammation is present at the cornea } \\
\text { peripheral to the bonded perforated cut and } \\
\text { limbus at this side. }\end{array}$ \\
\hline & $\mathrm{H}$ & Experimental failure & Experimental failure \\
\hline
\end{tabular}


Table 2 (Continued).

\begin{tabular}{|c|c|c|c|}
\hline Action & Piglet & OCT & Histology \\
\hline \multirow{8}{*}{$\begin{array}{l}\text { Group-III control: } \\
\text { slanted cut, } \\
\text { no albumin, } \\
\text { no laser heating. }\end{array}$} & I & 1. Same as the OCT results for piglet $D$. & $1,2,4,5,6$. Similar to the histology for piglet $A$. \\
\hline & & 2. $20 \%$ to $30 \%$. & 3. Not seen. \\
\hline & & $\begin{array}{l}\text { 3. Same as the OCT results for piglet } \mathrm{D} \text {, with slight } \\
\text { bulging into the anterior chamber. }\end{array}$ & $\begin{array}{l}\text { There is adhesion between the inner surface of } \\
\text { the scar and the anterior part of the iris. }\end{array}$ \\
\hline & $\mathrm{J}$ & 1,2 , 3. Same as the OCT results for piglet I, & Same as the histology results for piglet I. \\
\hline & $\mathrm{K}$ & $1,2,3$. Same as the OCT results for piglet I. & Same as the histology results for piglet I. \\
\hline & & $\begin{array}{l}\text { Massive excess hyperreflective tissue bulging into } \\
\text { the anterior chamber, anterior to a separate defined } \\
\text { anterior chamber structure, separated from } \\
\text { the cornea by a thin membranous limit. }\end{array}$ & \\
\hline & $\mathrm{L}$ & 1 , 2. Same as the OCT results for piglet $I$. & $1,2,3,4,5$. Similar to the histology for piglet $A$. \\
\hline & & 3. Same as the OCT results for piglet $D$. & 6. Same as the histology results for piglet $D$. \\
\hline
\end{tabular}

mild folding. A stromal thin scar was seen, extending from the epithelium to the endothelium with mild hypercellularity and microneovascularization (observed, but not shown). The Descemet's membrane was disrupted and the gap between its edges was occupied by a triangular-shaped scarring with the base toward the inner surface of the cornea.

\subsubsection{Welded group}

The histopathology and the OCT images shown in Fig. 3 for piglet $\mathrm{G}$ are those of an incision that was laser welded, using the slanted incision-zigzag heating protocol.

Most of the findings were similar to the ones of the soldered group (see above). Here, again, a completely closed incision is shown and the intact corneal shape indicates that the welding protocol did not cause a change in corneal shape. Some hyperreflectivity was noted on the outer layer of the cornea in the OCT images, which could be an indication of minor thermal damage. In the histopathology, a well-bonded incision was observed. In one sample, the cornea slightly bulged into the anterior chamber, and in another a possible minor wound sliding was detected.

\subsubsection{Control group}

The OCT image of the eye presented in Fig. 3 for piglet K (control group) shows an intact corneal shape that would indicate no detectable optical aberrations due to the protocol and a completely closed incision. Other findings were hyperreflectivity at the cut, some hyperreflectivity on the outer layer of the cornea, as well as some hyperreflective globular mass at the Descemet's membrane, even though no laser heating was applied. The histopathology image showed a well-sealed cut. A hyperreflective anterior stroma, encompassing roughly the outer $30 \%$ to $70 \%$ of the incision length, was seen, bulging into the anterior chamber. A measurable area of the posterior stromal hyperreflectivity near endothelial side of the wound was detected.
Figure 3 shows corneal images of three piglets, each representing one of the three groups. We notice that the radii of curvature of all the corneas, as determined by OCT and histopathology measurements, were similar in these groups. The results were similar to the ones observed in eyes extracted from pigs that were not processed in any way (including incising).

\section{Discussion}

Eyes that underwent corneal surgery must be able to withstand burst pressures that can be developed in various physiological responses, such as sneezing or coughing. As mentioned, the acceptable $P_{\mathrm{B}}$ for bonded corneal incisions is at least $80 \mathrm{mmHg}{ }^{36,37,41}$ The first aim of this work is to achieve this, or higher $P_{\mathrm{B}}$, immediately after the surgery. Toward this goal, we tested different irradiation and incision protocols. These included the vertical incision-linear heating, the vertical incision-zigzag heating, and the slanted incision-zigzag heating protocols, as presented in Table 1 .

It can be observed in Table 1 that in the ex vivo experiments the slanted incision-zigzag heating protocol generated nearly a 10 -fold higher $P_{\mathrm{B}}$ than the generally acceptable one. This is probably because the spreading of the albumin on a larger area and because the laser heating was done on this larger area (on the surface and in the bulk). The vertical incision-linear heating and the vertical heating-zig zag protocols generated $P_{\mathrm{B}}$ that was still higher than the quoted minimally acceptable value of $P_{\mathrm{B}}{ }^{36,37,41}$

We assumed that the same $P_{\mathrm{B}}$ values will apply for the in vivo cases. It is important to notice that the incisions in the control group eventually closed since these incisions are selfsealing. However, as mentioned, with no laser bonding the $P_{\mathrm{B}}$ was only $30 \mathrm{mmHg}$, which is far too low. Therefore, only the soldered incisions achieved a water-tight seal of very high strength, immediately after surgery, whereas the welded or nonheated incisions did not.

Though a more thorough comparison may be needed, the bonding quality obtained with the $1.9-\mu \mathrm{m}$ laser is better than the one obtained with a $\mathrm{CO}_{2}$ laser. ${ }^{29}$ This is most probably due 
to the difference in penetration depths and a more uniform heating along the depth of the incision.

We also note the relatively large standard deviation of the $P_{\mathrm{B}}$ results (Table 1). Our initial investigations traced this to how accurately the sample was cut. Introduction of "knifes" better than the standard scalpels may be needed. Femtosecond lasers were recently successfully adapted for ophthalmic laser surgery. ${ }^{42}$ This advancement has the potential to increase the reproducibility in the $P_{\mathrm{B}}$ results.

The in vivo experiments were performed under the assumption that the same immediate $P_{\mathrm{B}}$ would be maintained if the irradiation protocol of the optimized ex vivo experiments is repeated. The aim in the in vivo experiments was not to observe $P_{\mathrm{B}}$ but to observe the corneal shape, after the weekslong recovery period.

We believe that, as in any change in stromal integrity, there should be some change in the curvature of the cornea. The quantification of the degree of corneal distortion was not performed, using corneal topographic scans, as it was not available to us. We therefore attempted to qualitatively assess the change using both OCT and histopathology measurements (e.g., Fig. 3). We found that the histology and the OCT findings in corneas reported in Table 2 are quite similar for the three study groups. These showed complete closure and negligible change in corneal shape. From this, we conclude that the optical aberrations generated by this procedure should be negligible.

The OCT and histopathology measurements also revealed that the differences in the outcome were mostly in the area of the posterior stromal hyperreflectivity, near the endothelial side of either wound. In the soldered group it was small, whereas it slightly increased in the welded group and further slightly increased in the control group. Yet, all these changes were small. We note the overall similarity in all the images we obtained, regardless of the group to which they belonged. This similarity between the groups suggests that if there was thermal damage in the welding and soldering samples, it was insignificant.

We observed inflammation only in one in vivo eye out of the 12 quoted. This inflammatory reaction may be attributed to an abnormal recovery and not to the surgical protocol itself.

The outcome of this work is a demonstration that laser-soldering of corneas is feasible and that it generates an immediate and high burst-pressure bond. This should be of benefit to future corneal surgical protocols. Perpendicular incisions should be used for corneal transplantation, and we showed that the acceptable $P_{\mathrm{B}}$ has been achieved in this case. As for cataract surgery, it can benefit from the slanted incision-zigzag heating protocol, due to the exceptionally high bonding strength obtained with this improved method of corneal incision-closure.

Some of the advantages of laser soldering of corneal incisions, compared to suturing, include the following. (1) Immediate closure of nonpenetrating corneal wounds, such as in deep anterior lamellar keratoplasty. (2) Negligible optical aberrations and very little scarring were observed, thus demonstrating an optically transparent tissue. This will be beneficial for reducing postsurgical complications such as astigmatism. (3) In the case of corneal transplantation, a much easier and faster closure of a full 360-deg corneal graft has the potential to be obtained. (4) Reduction of the postoperative foreign body (sutures and stitches) sensation and discomfort. ${ }^{43}$ (5) Sealing of miniature cuts that may appear in the cornea as a result of a trauma. Such cuts are extremely hard to close, so the surgeons are relying on the corneal self-healing while accepting the deformations that are generated in the curvature of the cornea as a result.

All these important advantages could make laser soldering a very promising technique for various types of corneal surgery.

\section{Conclusions}

Laser bonding of incisions was successfully demonstrated by several groups worldwide. However, it has not yet made a clinical impact.

We developed a fiber-optic temperature-controlled lasersoldering system for bonding corneal incisions, using albumin as a solder. The use of visible and mid-IR fibers made it possible to accurately monitor and control the temperature of each bonded spot on an incision and to do it rapidly. We used this system for bonding either vertical incisions or slanted incisions in corneas of piglets. A strong and watertight bonding was obtained immediately in both procedures. We did not observe a change in the corneal shape if the eye was soldered, welded, or left to self-heal.

The skill required to operate the laser soldering system is significantly lower than the one needed for suturing by an experienced surgeon. This is a noncontact method and a surgeon only needs to maintain the handpiece at a set distance above the cut and slowly move the distal end along the incision.

Future experiments will focus on corneal transplantation in large pigs, and the success in those experiments will enable us to start human clinical trials. We hope that in the future, laser soldering could substitute suturing in corneal surgery.

\section{Disclosures}

The authors have no conflicting interests to declare.

\section{Acknowledgments}

This work was funded by the Israeli Ministry of Science Grant Nos. 3-10856, 3-15627, and by the Pazi Foundation Grant No. ID22-2018.

\section{References}

1. J. W. Allen et al., "Intracorporeal suturing and knot tying broadens the clinical applicability of laparoscopy," J. Soc. Laparoendosc. Surg. 7(2), 137-140 (2003)

2. I. Nisky et al., "Teleoperated versus open needle driving: kinematic analysis of experienced surgeons and novice users," in IEEE Int. Conf. Rob. and Autom., pp. 5371-5377 (2015).

3. M. Severin and K.-U. Bartz-Schmidt, Penetrating Keratoplasty: Diagnosis and Treatment of Postoperative Complications, Springer, Berlin, Heidelberg (2000).

4. J. Hjortdal et al., "Influence of suture regularity on corneal astigmatism after penetrating keratoplasty," Acta Ophthalmol. 89(5), 412-416 (2011).

5. L. S. Bass and M. R. Treat, "Laser tissue welding: a comprehensive review of current and future clinical applications," Lasers Surg. Med. 17(4), 315-349 (1995).

6. R. Rasier et al., "Corneal tissue welding with infrared laser irradiation after clear corneal incision," Cornea 29(9), 985-990 (2010).

7. P. K. Vaddavalli and S. H. Yoo, "Technology needs for corneal transplant surgery," Proc. SPIE 7885, 788502 (2011).

8. R. Pini, L. Menabuoni, and L. Starnotti, "First application of laser welding in clinical transplantation of the cornea," Proc. SPIE 4244, 266-271 (2001).

9. L. Buzzonetti et al., "Laser welding in penetrating keratoplasty and cataract surgery in pediatric patients: early results," J. Cataract Refract. Surg. 39(12), 1829-1834 (2013). 
10. A. Canovetti et al., "Laser-assisted penetrating keratoplasty: 1-year results in patients using a laser-welded anvil-profiled graft," Am. J. Ophthalmol. 158(4), 664-670.e2 (2014).

11. A. Lauto et al., "Carotid artery anastomosis with albumin solder and near infrared lasers: a comparative study," Lasers Surg. Med. 28(1), 50-55 (2001).

12. A. Lauto et al., "In vitro and in vivo tissue repair with laser-activated chitosan adhesive," Lasers Surg. Med. 39(1), 19-27 (2007).

13. A. P. Duarte et al., "Surgical adhesives: systematic review of the main types and development forecast," Prog. Polym. Sci. 37(8), 1031-1050 (2012).

14. F. Rossi et al., "Laser assisted robotic surgery in cornea transplantation," Proc. SPIE 10056, 100560T (2017).

15. G. Esposito et al., "In vivo laser assisted microvascular repair and endto-end anastomosis by means of indocyanine green-infused chitosan patches: a pilot study," Lasers Surg. Med. 45(5), 318-325 (2013).

16. E. E. Verter et al., "Light-initiated bonding of amniotic membrane to cornea," Invest. Ophthalmol. Visual Sci. 52(13), 9470-9477 (2011).

17. T. Wang et al., "Photochemical cross-linking for penetrating corneal wound closure in enucleated porcine eyes," Curr. Eye Res. 42(11), 1413-1419 (2017).

18. F. Rossi et al., "Laser tissue welding in ophthalmic surgery," $J$. Biophotonics 1(4), 331-342 (2008).

19. R. W. Redmond and I. E. Kochevar, "Medical applications of rose Bengal- and riboflavin-photosensitized protein crosslinking," Photochem. Photobiol. 95(5), 1097-1115 (2019).

20. I. Gabay et al., "Temperature-controlled two-wavelength laser soldering of tissues," Lasers Surg. Med. 43(9), 907-913 (2011).

21. I. Gabay, "Temperature controlled laser bonding of corneal incisions using a single infrared fiber system," $\mathrm{PhD}$ Thesis, Tel Aviv University, Israel (2014).

22. A. M. Sagi-Dolev et al., "Heating of biological tissue by laser irradiation: temperature distribution during laser ablation," Opt. Eng. 31(7), 1425-1431 (1992).

23. A. Barak et al., "Temperature-controlled $\mathrm{CO}_{2}$ laser tissue welding of ocular tissues," Proc. SPIE 2971, 103-105 (1997).

24. I. Gabay et al., "Closure of incision in cataract surgery in-vivo using a temperature controlled laser soldering system based on a $1.9 \mu \mathrm{m}$ semiconductor laser," Proc. SPIE 9702, 97020B (2016).

25. I. Gabay et al., "Bonding surgical incisions using a temperaturecontrolled laser system based on a single infrared fiber," J. Biomed. Opt. 18(11), 111416 (2013).

26. E. Strassmann et al., "Laser soldering of the cornea in a rabbit model using a controlled-temperature $\mathrm{CO}_{2}$ laser system," Proc. SPIE $\mathbf{4 2 4 4}$ 253-265 (2001).

27. Y. Porat et al., "Optical coherence tomography (OCT) in laser tissue bonding of incisions in the cornea," Proc. SPIE 9317, 93170M (2015).
28. G. Norman et al., "In vitro conjunctival incision repair by temperaturecontrolled laser soldering," J. Biomed. Opt. 14(6), 064016 (2009).

29. D. Levanon, A. Katzir, and A. Ravid, "A scanning electron microscopy study of $\mathrm{CO}_{2}$ laser-albumin soldering in the rabbit model," Photomed. Laser Surg. 22(6), 461-469 (2004).

30. D. Spector et al., "In vitro large diameter bowel anastomosis using a temperature controlled laser tissue soldering system and albumin stent," Lasers Surg. Med. 41(7), 504-508 (2009).

31. B. Forer et al., " $\mathrm{CO}_{2}$ laser fascia to Dura soldering for pig dural defect reconstruction," Skull Base 17(1), 017-023 (2007).

32. D. Yafit et al., "Laser soldering of cartilage graft interposed into a tracheal incision in a porcine model," Laryngoscope 129(1), 58-62 (2019).

33. D. Simhon et al., "Immediate tight sealing of skin incisions using an innovative temperature-controlled laser soldering device: in vivo study in porcine skin," Ann. Surg. 245(2), 206-213 (2007).

34. D. Simhon et al., "Temperature-controlled laser-soldering system and its clinical application for bonding skin incisions," J. Biomed. Opt. 20(12), 128002 (2015).

35. S. Basov et al., "Robot-assisted laser tissue soldering system," Biomed. Opt. Express 9(11), 5635-5644 (2018).

36. A. M. Oelker and M. W. Grinstaff, "Ophthalmic adhesives: a materials chemistry perspective," J. Mater. Chem. 18(22), 2521-2536 (2008).

37. G. Trujillo-de Santiago et al., "Ocular adhesives: design, chemistry, crosslinking mechanisms, and applications," Biomaterials 197, 345367 (2019).

38. E. Strassmann et al., " $\mathrm{CO}_{2}$ laser welding of corneal cuts with albumin solder using radiometric temperature control," Ophthalmic Res. 50(3), 174-179 (2013).

39. P. Holl et al., "Recent advances in power scaling of GaSb-based semiconductor disk lasers," IEEE J. Sel. Top. Quantum Electron. 21(6), 324-335 (2015).

40. F. Rossi et al., "Experimental study on the healing process following laser welding of the cornea," J. Biomed. Opt. 10(2), 024004 (2005).

41. J. Shahbazi et al., "Sutureless sealing of penetrating corneal wounds using a laser-activated thin film adhesive," Lasers Surg. Med. 43(6), 490-498 (2011).

42. A. Canovetti et al., "Anvil-profiled penetrating keratoplasty: load resistance evaluation," Biomech. Model. Mechanobiol. 18(2), 319-325 (2019).

43. L. Menabuoni et al., "Laser-assisted corneal welding in cataract surgery: retrospective study," J. Cataract Refract. Surg. 33(9), 1608-1612 (2007).

Biographies of the authors are not available. 